\title{
Influence of Methacrylic-Acrylic Copolymer Composition on Plasticiser-free Optode Films for pH Sensors
}

\author{
Lee Yook Heng*, Teh Huey Fang, Loh Han Chern and Musa Ahmad \\ School of Chemical Sciences \& Food Technology, Faculty of Science and Technology, Universiti \\ Kebangsaan Malaysia, 43600 Bangi, Selangor, Malaysia. \\ * Author to whom correspondence should be addressed.
}

Received: 7 March 2003 / Accepted: 24 March 2003 / Published: 27 March 2003

\begin{abstract}
In this work we have examined the use of plasticiser-free polymeric films incorporating a proton selective chromoionophore for optical $\mathrm{pH}$ sensor. Four types of methacrylic-acrylic copolymers containing different compositions of n-butyl acrylate (nBA) and methyl methacrylate (MMA) were synthesised for use as optical sensor films. The copolymers were mixed with appropriate amounts of chromoionophore (ETH5294) and a lipophilic salt before spin coated on glass slides to form films for the evaluation of $\mathrm{pH}$ response using spectrophotometry. Co-polymer films with high nBA content gave good response and the response time depended on the film thickness. A preliminary evaluation of the optical films of high nBA content with pHs from 2 - 14 showed distinguishable responses from $\mathrm{pH} 5$ - 9. However, the adhesion of the $\mathrm{pH}$ sensitive film was good for copolymers with higher content of MMA but not for films with high nBA.
\end{abstract}

Key words: Optical pH sensor, Methacrylic-acrylic films, Chromoionophore, Plasticiserfree.

\section{Introduction}

Polymers have been given both theoretical interest and practical application in the field of sensor technology in the last decade. They can be used for very different purposes and may offer unique applications. Polymers offer many advantages for sensor technologies: they are relatively low cost, their fabrication techniques are simple and can be deposited on various types of substrates. The established molecular structure of the polymers also allows some possibilities to build in side-chains, charged or neutral moieties, and even grains of specific properties into the bulk material or on its 
surface region, thus enables films to be produced with various physical and chemical properties for a desirable sensing behaviour ${ }^{(1)}$. The various sensors applying sensing polymers can be used for physical measurements such as temperature, mechanical properties (touch switch devices, deformation sensors, pressure sensors, etc.), acoustic properties (microphones, ultrasonic sensors, etc.), infra-red radiation, relative humidity and physical gas sensing. Polymers can be used as membranes that are sensitive and specific to the detection of ion concentrations, also in special sensors for medical and biology, e.g. enzyme and immunosensors ${ }^{(1)}$.

Optodes or optical sensing devices have been developed for almost 30 years. Chemical sensors based on optical transduction are used in the analysis of an infinite broad spectrum of analytes including the detection of charged compounds. Several properties are important for a successful application of optical transduction, these include the intrinsic optical effects and inherent optical characteristics of a specific analyte. A host molecule or indicator that responds to the analyte to yield an optical signal is usually employed in optical sensor ${ }^{(2)}$.

Optical sensor based on plasticised PVC membranes to detect ions have been long developed. However, there have been problems mainly due to the poor membrane adhesion and drift of the sensor response resulted from leaching of plasticiser used in the membrane formulation. A high plasticiser content in the membrane is also known to make the polymer less biocompatible ${ }^{(3)}$. Thus, a polymer requires no plasticiser for an optode for ion detection will be useful.

Recently, the acrylate family of polymers has been used successfully as membranes for potentiometric ion sensors $^{(4-6)}$ without the use of a plasticiser. Since then, the application of this class of plasticiser-free polymers has been extended to optical ion sensors, particularly membranes based on cross-linked dodecyl acrylate ${ }^{(7)}$ and decyl methacrylate ${ }^{(8)}$. In this work, we have examined the copolymers of methacrylate-acrylate as plasticiser-free films for the optical detection of $\mathrm{pH}$. An optical sensor was constructed by incorporating a chromoionophore that is selective to $\mathrm{H}^{+}$into the plasticiserfree methacrylic-acrylic films. The effect of the copolymer composition on the behaviour of the optical sensor was also investigated.

\section{Experimental}

Materials: Methyl methacrylate (MMA), n-butyl acrylate (nBA), sodium tetrakis(3,5bis(trifluoromethyl)phenyl]borate (NaTFPB), chromoinophore ETH5294, chlorides of potassium, sodium and lithium (all are analytical grade) from Fluka, chloroform from Merck, petroleum ether 60$80^{\circ} \mathrm{C}$ from Fisher and sodium hydroxide pellets, dichloromethane from J.T. Baker. Standard solutions were prepared from salts and doubly deionized water.

Synthesis of polymers: Copolymers of MMA and nBA were prepared according to reported procedure $^{(4)}$. Solution polymerisation was used. The compositions of the copolymers based on monomer used are shown in Table 1.

Preparation of optode: Optode films were prepared from a mixture of $0.05 \mathrm{~g}$ of copolymer methacrylic-acrylic, $0.002 \mathrm{~g}$ of NaTFPB and $0.001 \mathrm{~g}$ chromoionophore ETH5429 in 500 $\mu \mathrm{L}$ chloroform. Approximately $100 \mu \mathrm{L}$ of the solution was cast onto a glass slide $(9 \mathrm{~mm}$ x $26 \mathrm{~mm})$ by using a spin coater (Chemat Technology). Before the mixture was spin coated, the glass slide was cleaned with acetone 
and dried. After coating, the optode was left to dry at room temperature for $24 \mathrm{~h}$. When dried, the optode film gave a blue colour indicating the presence of the protonated form of ETH5294.

The thickness of the copolymer film was estimated from calculation based on the weight of polymer used, the area coated and the density, which assumed to be $1 \mathrm{gmL}^{-1}$ because most acrylic based polymers have density close to that value ${ }^{(9)}$. A relationship of the weight of polymer used in coating $(0.04-0.06 \mathrm{~g})$ and thickness of film obtained was established with this method. The variation in thickness (D, by calculation) and the weight (W) of copolymers spin coated (in a fixed volume of solvent) followed a linear relationship $\left(\mathrm{W}=144.7 \mathrm{D}-1.73, \mathrm{R}^{2}=0.70, \mathrm{n}=11\right)$. This procedure was used to calculate the thickness for all four copolymer films. The estimated thickness was then employed in the study of adhesion of the copolymer films on glass slide substrate. The optode films prepared from each copolymer were then immersed in TrisHCl buffer $(\mathrm{pH} 7)$ and the time taken for the film completely detached from the slide was noted.

Table 1. The composition of copolymers used for optode studies.

\begin{tabular}{|c|c|c|}
\hline Polymer & $\begin{array}{c}\text { MMA } \\
\text { (mol fraction) }\end{array}$ & $\begin{array}{c}\text { nBA } \\
\text { (mol fraction) }\end{array}$ \\
\hline MB19 & 0.11 & 0.89 \\
\hline MB28 & 0.20 & 0.80 \\
\hline MB37 & 0.30 & 0.70 \\
\hline MB46 & 0.44 & 0.56 \\
\hline
\end{tabular}

Spectrophotometric studies of optode films: Spectrophotometric studies on optode films were performed with a $1-\mathrm{cm}$ quartz cell mounted in a Cary-100 UV-Visible spectrophotometer. TrisHCl buffer of various pHs were used as standard solutions to test the optode response. Absorption spectra were recorded between 350 to $750 \mathrm{~nm}$ at $25 \pm 1^{\circ} \mathrm{C}$ at an interval of $2 \mathrm{~min}$. The equilibrium response of the optode was reached when the absorption value remained constant for $10 \mathrm{~min}$.

Before the $\mathrm{pH}$ response of the optode was assessed, the chromoionophore was converted to the unprotonated red form by using $6 \mathrm{M}$ of $\mathrm{NaOH}$. The red colour film was then employed in measuring $\mathrm{pH}$ responses from $\mathrm{pH}$ range 2 - 10. After each measurement, the optode film was again converted to the unprotonated form before measurements on other $\mathrm{pHs}$ were carried out.

\section{Results and Discussion}

Effect of film thickness and copolymer types on adhesion of optode films

The adhesion characteristic of the four types of copolymer on glass slide was examined by the time taken for a copolymer film to be detached after exposure to a $\mathrm{pH}$ buffer. In general, the type of copolymer seems to influence heavily the time taken for a film to be detached from the glass substrate. From Figure 1, the copolymer MB19 took about 10 - $20 \mathrm{~min}$ to detach from the glass slide whilst the other copolymers required up to 10 hours before the film began to peel off. The high amount of nBA in the copolymer MB19 obviously caused the polymer to be very soft and weak, this may reduce the adhesion on the glass substrate. 


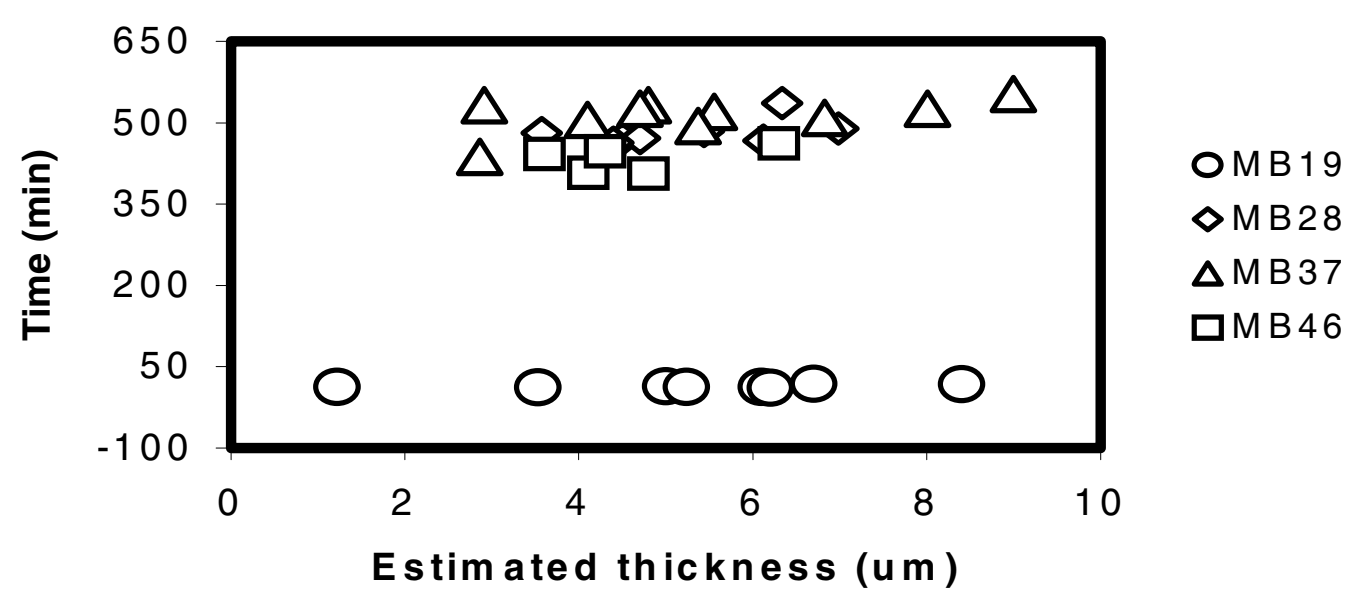

Figure 1. The time taken for different types of copolymer films to detach from the glass slides after immersed in Tris $(\mathrm{HCl})$ buffer $\mathrm{pH}=7$ with respect to the calculated thickness.

Clearly, MB19 is not desirable for use in optical sensor because the lifetime of the sensor will be short due to poor adhesion on glass surface. However, the softness and elasticity of the non-plasticised based polymers is important in providing flexibility to the chromoionophore in the extraction of $\mathrm{H}^{+}$by complexation processes $^{(6)}$. For MB37 and MB46, which expected to have much higher content of MMA, better adhesion property was observed but unfortunately they did not give appreciable response to changes in $\mathrm{pH}$ when the ETH5294 was entrapped in the films together with NaTFPB. These copolymers are 'hard' and have much higher glass transition temperatures ${ }^{(5)}$. The only copolymer among all four types that could be used for optode study is therefore MB28 that demonstrated both good adhesion and sufficient softness. This copolymer was subsequently used for $\mathrm{H}^{+}$selective optode study in this work.

\section{Spectrophotometric study of $\mathrm{H}^{+}$-selective optode film}

In a plasticised and bulk optode film, the ions of interest are extracted into the plasticised polymeric membrane by mass transfer where the charge created will cause a change in the optical signal of the extracting species (e.g. the chromoionophore), which is detected by means of absorption or fluorescence ${ }^{(10,11)}$. In the case of chromoionophore that extract $\mathrm{H}^{+}$, e.g. ETH5294, it can exist in the film in a neutral or basic form. The neutral chromoionophore becomes positively charged when protonated by extracting $\mathrm{H}^{+(10)}$. The overall neutrality of the optode film is compensated by the addition of a lipophilic salt such as NaTFPB. The processes occurring in an optode membrane may be described by the ion-exchange reaction between an aqueous (aq) sample and the organic (org) membrane phase (12).

$$
\mathrm{H}^{+}(\mathrm{aq})+\mathrm{C}_{\mathrm{m}}(\text { org })=\mathrm{HC}_{\mathrm{m}}^{+}(\text {org })
$$

If the ratio of the concentration of the deprotonated chromoionophore to that of its total concentration $\left(\mathrm{C}_{\mathrm{mT}}\right)$ present in the membrane phase is denoted by $\alpha$, the degree of deprotonation, is then: 


$$
\left[\mathrm{C}_{\mathrm{m}}\right]=\alpha \cdot \mathrm{C}_{\mathrm{m}} \mathrm{T} \text { and }\left[\mathrm{HC}_{\mathrm{m}}^{+}\right]=(1-\alpha) \mathrm{C}_{\mathrm{m}} \mathrm{T}
$$

and this can be related to the absorbance, A, if spectrophotometric measurements are carried out.

$$
\alpha=\left(\mathrm{A}-\mathrm{A}_{\mathrm{o}}\right) /\left(\mathrm{A}_{1}-\mathrm{A}_{\mathrm{o}}\right)
$$

where $A_{1}$ and $A_{0}$ are the limiting absorbance values for $\alpha=1$ (chromoionophore completely deprotonated) and $\alpha=0$ (totally protonated), respectively.

For the process of deprotonation-protonation of an optode film containing ETH5294, deprotonation in $6 \mathrm{M} \mathrm{NaOH}$ leads to the increase in the neutral form, which has a maximum absorption in $543 \mathrm{~nm}$ whilst at the same time the positively charged form decreases as shown by a gradual reduction in maximum absorption at 610 and $660 \mathrm{~nm}$ (Figure 2). The isobestic point of the chromoionophore represents the transformation of the two forms ${ }^{(13)}$.

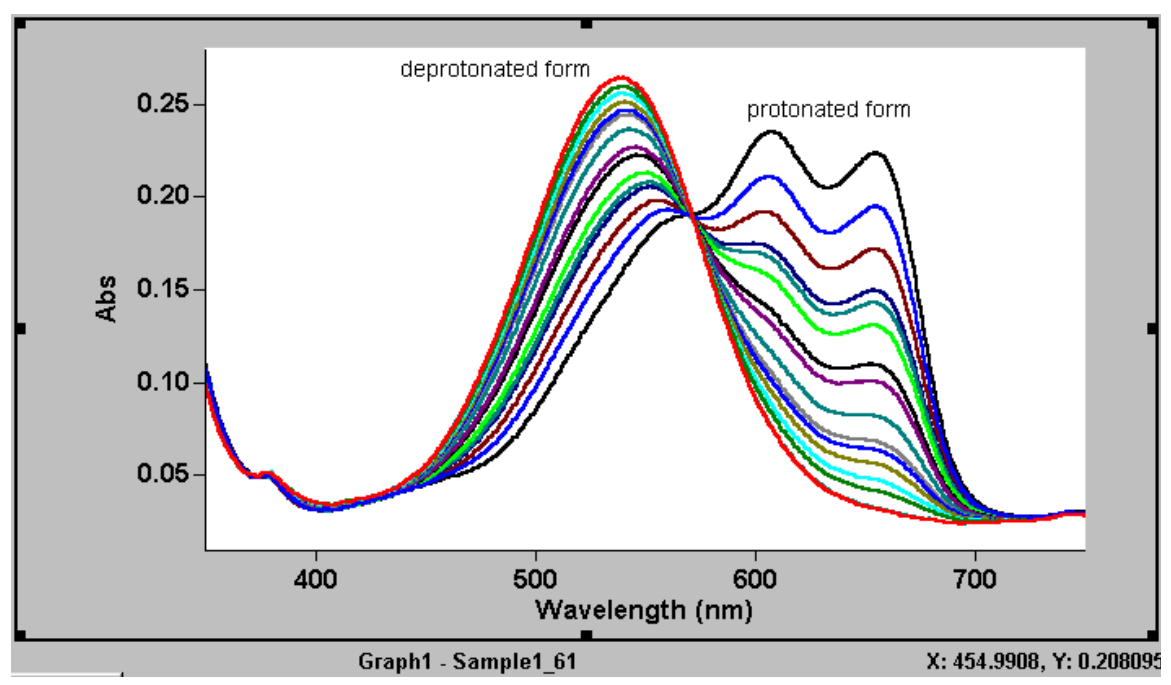

Figure 2. The absorption behaviour of a MB28 optode film containing chromoionophore ETH5294 and NaTFPB in $6 \mathrm{M}$ of $\mathrm{NaOH}$ showing conversion of the initial fully protonated form to the completely deprotonated form.

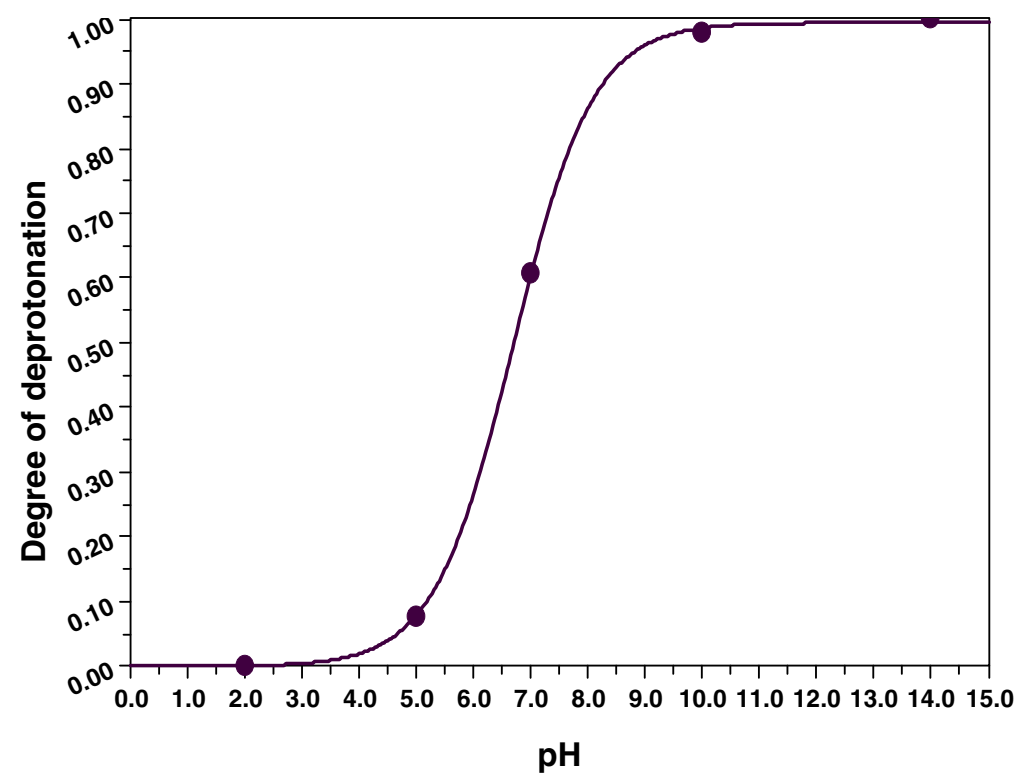

Figure 3. The response $(\alpha)$ for an optode with a MB28 film exposed to $\mathrm{pH}$ 2-14. 
From Figure 2, by recording the absorption at $543 \mathrm{~nm}$ in $6 \mathrm{M} \mathrm{NaOH}(\alpha=1)$, in $1 \mathrm{M} \mathrm{HCl}(\alpha=0)$ and equilibrium absorption at a particular $\mathrm{pH}$, the value of $\alpha$ for a certain $\mathrm{pH}$ can be determined. Thus, a plot of the $\alpha$ values against the $\mathrm{pHs}$ yielded a reponse curve for the $\mathrm{H}^{+}$- selective optode. The response is linear from approximately $\mathrm{pH} 5-8$ as shown in Figure 3. This profile of $\mathrm{pH}$ response is similar to that reported by Bakker et al using the same chromoionophore but in a plasticised PVC membrane ${ }^{(14)}$.

\section{Selectivity of Optode Films}

While the sensitivity of an optode membrane is dependent on sufficient concentration of chromoionophore presence in the membrane bulk ${ }^{(12)}$, the selectivity of the optode towards $\mathrm{H}^{+}$against other ions, especially cations is very much an intrinsic property of the chromoionophore and the amount of lipophilic anions incorporated into the membrane. Adding NaTFPB (ETH5294:NaTFPB = 2:1) will control the total concentration of ions within the optode membrane. The response towards $\mathrm{H}^{+}$ at $\mathrm{pH}=7$ for optode membranes with ETH5294 as chromoionophore in the presence of $0.1 \mathrm{M}$ of sodium, potassium or lithium ions showed that the degrees of deprotonation, $\alpha$, were only changed by +0.04 to -0.03 . This is small when compared to a typical change of $\alpha$ by one unit change of $\mathrm{pH}$, which is approximately 0.3 . Thus, it can be concluded that other cations such as $\mathrm{Na}, \mathrm{K}$ and $\mathrm{Li}$ did not exert any clear interference on the $\mathrm{H}^{+}$response of the optode and the sensor is selective to $\mathrm{H}^{+}$ions.

\section{The Response Time of the $H^{+}$- Selective Optode}

The chromoionophore needs high degree of mobility in the optode membrane because of the rather diffusion-limited response ${ }^{(2)}$. Different compositions of the MMA and nBA gave different response behaviour and response times for the films studied, e.g. MB28 cf. MB19 (Figure 4). Optode films with high nBA content, e.g. MB19 gave faster response when compared to higher MMA copolymer films such as MB28. Film with even higher MMA content such as MB46 demonstrated very slow and only a slight response to $\mathrm{pH}$ change. MB19 and MB28 are clear plastic-like copolymers while MB46 is a glassy solid. In potentiometric sensors, the glass transition temperature of the membrane based on plasticiser-free methacrylic-acrylic copolymers is known to affect the ion response of the sensors. Low glass transition temperature means a flexible polymer matrix and this allows the ionophore to exhibit its complexation behaviour once entrapped or covalently attached to the membrane ${ }^{(5,6)}$. This may explain the poor response of optode MB46 film where the glass transition temperature of this copolymer has been reported to be higher ${ }^{(5)}$. Furthermore, the inflexibility of the copolymer MB46 limits the partition and diffusion of the chromoionophore within the copolymer film. This will resulted in more time taken for protonation and deprotonation of the chromoionophore (Figure 4).

Apart from the copolymer composition, another parameter that affects the response time is the thickness of the optode film. From Figure 4, in order to reach equilibrium response quickly, bulk optode membranes need to be thin i.e. $<4 \mu \mathrm{m}$. The response time increases with the square of the membrane thickness. Thicker polymeric film has a much longer equilibrium response time than a thinner film. MB28 optode films with reduction in thickness clearly reached equilibrium response in a shorter time than thicker film. For a MB28 film to have a response time of approximately 10 minutes, 
a thickness of $1 \mu \mathrm{m}$ is necessary (Figure 4). Therefore, the thickness of the copolymer films is an essential parameter for controlling the response time of an optical sensor based on methacrylic-acrylic films apart from the chemical composition of the copolymer.
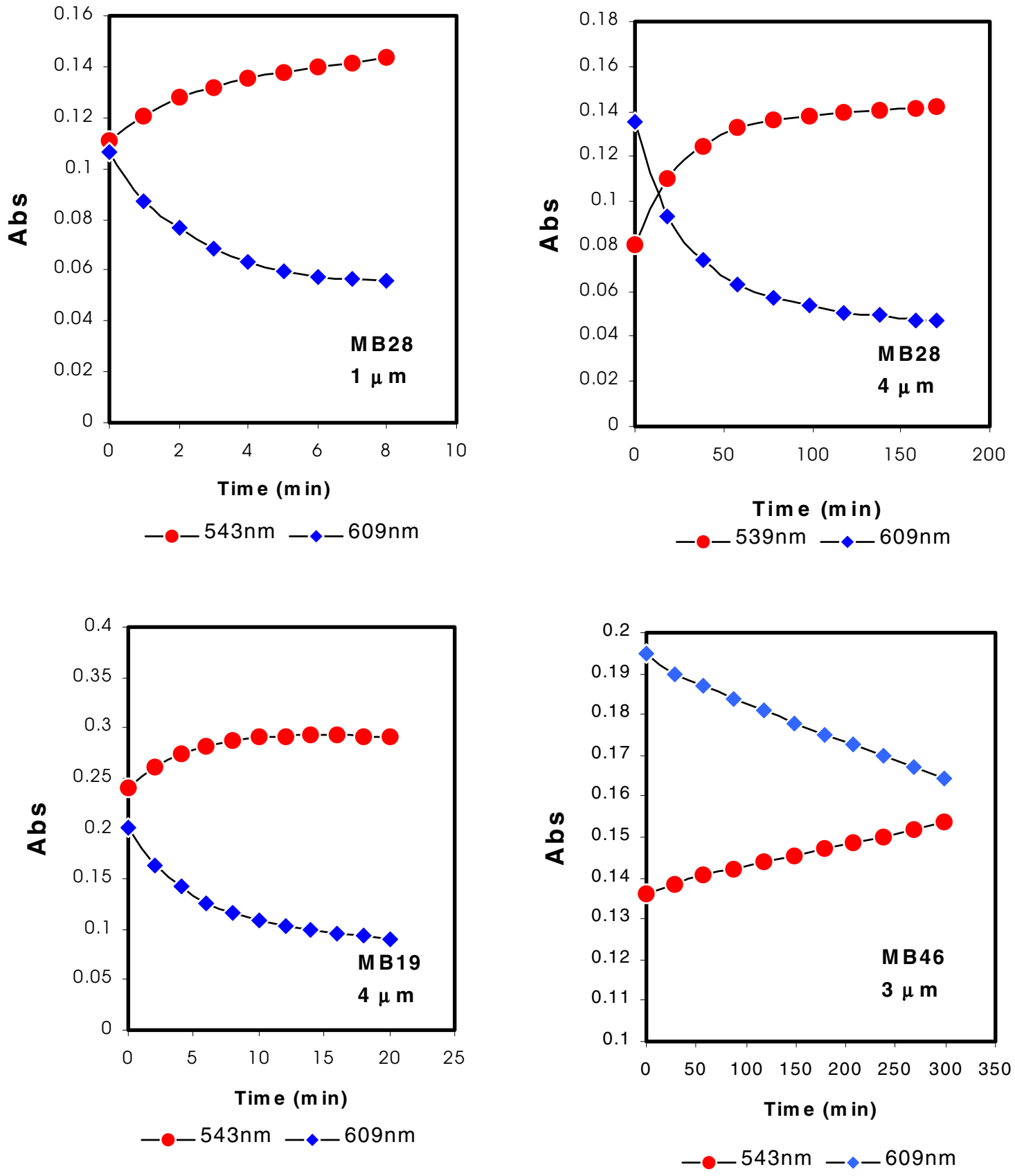

Figure 4. The response of various copolymer optode films with different thickness measured at two wavelengths after exposure to $6 \mathrm{M}$ of $\mathrm{NaOH}$.

\section{Conclusion}

Although the study carried out here on $\mathrm{H}^{+}$-selective optode based on plasticiser-free methacrylicacrylic films is brief and preliminary, the results showed that methacrylic-acrylic films without 
plasticiser could still be functional as ion-selective optode, especially when the copolymer composition is mainly n-butyl acrylate. This observation is in agreement with a number of ion sensors employing this type of copolymer matrices but based on potentiometric detection. The copolymer composition not only has effects on the adhesion property of the optode film but also the response time of the sensor.

\section{Acknowledgement}

Financial support through research grant IRPA 09-02-02-0136 for this work and a scholarship awarded to Loh Han Chern by the Malaysian National Science Fellowship is greatly acknowledged.

\section{References}

1. Gabor, H. Sensor Review 2000, 20, 98-105.

2. Ursula, E.S.K. Sensors and Actuators 1997, B38-39, 68-77.

3. Lindner, E.; Cosofret, V.V.; Ufer, S; Buck, R.P.; Kao, W.J.; Neuman, M.R.; Anderson, J.M. J. Biomedical Materials. Research. 1994, 28, 591-601.

4. Heng, L.Y.; Chern, L.H; Ahmad, M. Sensors 2002, 2, 339-346.

5. Heng, L.Y.; Hall, E. A. H., Anal. Chem. 2000, 42-51.

6. Heng, L.Y.; Hall, E.A.H. Electroanalysis. 2000, 12, 178-186.

7. Peper, S.; Tsagkatakis, I.; Bakker, E. Anal. Chim. Acta 2001, 442, 25-33.

8. Ambrose, T.M.; Meyerhoff, M.E. Anal. Chim. Acta. 1999, 378, 119-126.

9. Bandrup, J.; Immergut, E.H. Polymer Handbook, John Wiley \& Sons, 1989.

10. Eric, B.; Wilhelm, S. Anal. Chem. 1992, 64, 1805-1812.

11. Ursula, S.; Wilhelm, S.; Eric, B.; Markus, L.; Philippe, B. Sensors and Actuators 1993, B11, 1-8.

12. Wang, K.; Seiler, K.; Morff, W. E.; Spichger, U.E.; Simon, W.; Lindner, E.; Pungor, E. Analytical Sciences 1990, 6, 715-720.

13. Satoshi, O.; Peter, C. H.; Kurt, S.; Susie, S. S. T.; Werner, E. M.; Wilhelm, S. Anal. Chem. 1991, 63, 640-644.

14. Bakker, E.; Lerchi, M.; Rosatzin, T.; Rusterholz, B.; Simon, W. Anal. Chim. Acta. 1993, 278, 211 225.

Sample Availability: Available from the authors.

(C) 2003 by MDPI (http://www.mdpi.net). Reproduction is permitted for noncommercial purposes. 